\title{
Observation and manipulation of tris(dibenzoylmethanato)ruthenium molecules by low temperature scanning tunneling microscopy
}

\author{
L. Vernisse, O. Guillermet, J. Bonvoisin, and R. Coratger \\ CEMES, CNRS UPR 8011 et Université Paul Sabatier, \\ 29 rue J. Marvig, BP 94347, F-31055 Toulouse, France*
}

(Dated: September 24, 2013)

\begin{abstract}
The behaviour of tris(dibenzoylmethanato)ruthenium molecules has been studied using low temperature scanning tunneling microscopy after adsorption on $\mathrm{Ag}(111)$ and $\mathrm{Cu}(111)$. On both surfaces, the molecules present two adsorption geometries : a bi-lobed and a chiral threefold structure. This latter form can stand up to adopt a bi-lobed conformation which is oriented along the dense crystallographic directions. The molecular geometries after adsorption can be modified using the STM tip. In one of these modifications, a switch between two different conformations has been observed. The data recorded during the experiments show that this phenomenon is induced by the electric field in the STM junction.
\end{abstract}

Keywords: Scanning tunneling microscopy, UHV, low temperature, adsorption, ruthenium complexes, switch

\section{INTRODUCTION}

In molecular electronics, charge transfer in single molecules is of fundamental importance in the building of future active components [1]. Accordingly, a great number of investigations deals with the properties of molecules adsorbed on flat surfaces which can be used for this purpose. This interest is due to the fact that the behaviour of these molecules can be substantially changed when they are deposited on a substrate as compared to the properties observed in the gas phase. Indeed, the interactions with the surface significantly disrupts their electronic states which then couple each other $[2,3]$. Among these molecules, only a few examples deal with the study of mixed valence complexes $[4,5]$. These molecules are of peculiar interest since charge transfer between the redox centres offers the opportunity to perform charge engineering at the molecular scale. In this context, it is important to characterize single molecules since most of the usual characterisation techniques average their physical and chemical properties. This explains that in the recent years, scanning tunneling microscopy (STM) and its amazing resolution has largely been used to investigate a wide variety of molecules. These latter are then deposited on metal surfaces [6], semiconducting substrates $[7,8]$ or ultra-thin insulating layers [9] from crucibles or more simply from filaments heated by Joule effect. These constraints imply that the molecules should be able to support ultra high vacuum (UHV) conditions, evaporation process and adsorption on the substrate without any perturbations [10]. Then, their stability appears as a key point for in depth STM investigations. These conditions are generally fulfilled for planar molecules [11] but the situation is more complex for large $3 \mathrm{D}$ compounds which is generally the case for mixed valence complexes.

* vernisse@cemes.fr
Another point concerns the ability of these molecules to exhibit different switching states, characterized by different chemical and/or physical properties [12]. For mixed valence complexes, this is particularly important since the different oxidation states are linked to charge transfer through the molecule. In STM experiments, the switch is generally due to inelastic electrons tunneling through the junction $[13,14]$. One of the most striking examples concerns azobenzene derivatives adsorbed on noble $\mathrm{Au}(111)$ surfaces which can be switched between the cis and trans isomers as a function of tunneling current [14]. More rarely, the field generated by the applied bias voltage leads to conformational changes [15]. For molecular landers, these changes allow to manipulate the molecule on the surface because the electrical field induces a dipole in the molecular legs [16]. The molecular charge in mixed valence moieties is generally induced by sudden bias voltage variations [17] and then, one has to anticipate the possible resulting conformations. Thus, in accordance with the two points discussed above, mixed valence complexes should be first, easily deposited and then, manipulated with the tip if one is interested by their different charging states after adsorption.

In previous papers, we reported the first STM characterization of mononuclear ruthenium complexes with different ligands $[18,19]$. These molecules are of peculiar interest in the route towards the synthesis of larger entities devoted to charge transfer. We also showed that their evaporation on $\mathrm{Ag}(111)$ resulted in two different adsorption geometries, bi-lobed and threefold objects, the latter presenting two chiral structures.

In this article, we have particularly studied tipdependant modifications of the molecular geometry after adsorption of tris(dibenzoylmethanato)ruthenium $\left(\mathrm{Ru}(\mathrm{dbm})_{3}\right)$ on $\mathrm{Ag}(111)$ and $\mathrm{Cu}(111)$. On the two substrates, the adsorption geometries are the same. A first set of manipulations shows the transformation of a threefold object to a bi-lobed conformation. It is also demonstrated that the bi-lobed structure presents an unexpected property which consists in a switch between two 
different conformations controlled using the STM tip.

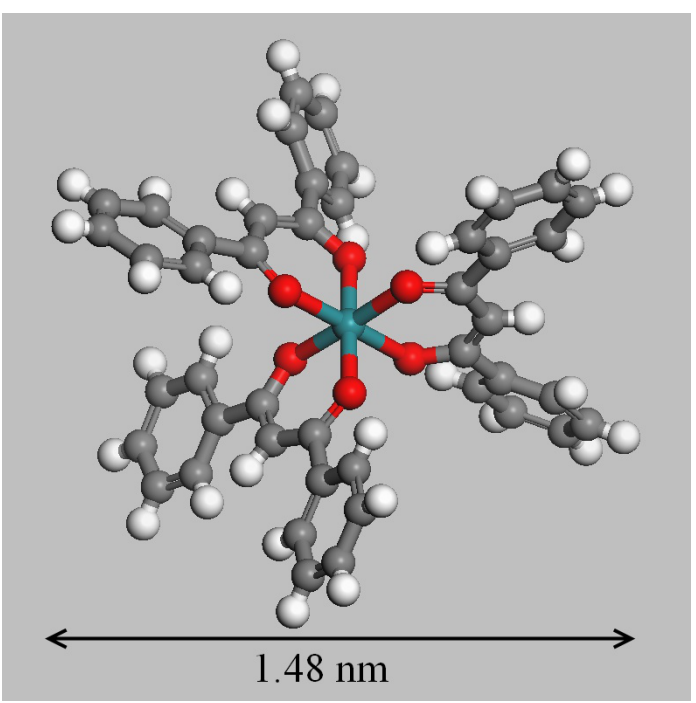

FIG. 1. Chemical structure of the $\mathrm{Ru}(\mathrm{dbm})_{3}$ molecule. Carbon atoms are in grey, hydrogen in white, oxygen in red. The central blue atom is ruthenium.

\section{EXPERIMENTAL DETAILS}

Scanning tunneling and atomic force microscopy experiments have been performed at liquid helium temperature ( $T=4,5 \mathrm{~K}$ ) on a commercial STM-AFM (LT Omicron) working with a base pressure of $2 \times 10^{-11}$ mbar. The $\mathrm{Ag}(111)$ and $\mathrm{Cu}(111)$ crystals are cleaned by repeated $\mathrm{Ar}^{+}$bombardment cycles at $E=600 \mathrm{eV}$ followed by annealing at $800 \mathrm{~K}$ for one hour. The $\mathrm{Ru}(\mathrm{dbm})_{3}$ molecule (Fig 1) is a three-dimensional object containing 85 atoms. The molecules are deposited on a $\mathrm{W}$ filament $0.15 \mathrm{~mm}$ in diameter. This filament is submitted to high temperature outgassing cycles before evaporation. In these manipulations, the substrate was held at liquid helium temperature and the molecules are directly evaporated on the STM head through a small hole $4 \mathrm{~mm}$ in diameter drilled in one of the windows of the He cryostat. The filament temperature is estimated to be $\sim 250{ }^{\circ} \mathrm{C}$. The small distance between the filament and the surface supports very small evaporation rates with typical values in the $1 / 1000$ monolayer range. These conditions are used for single molecule experiments in which the thermal diffusion is inhibited. STM tips made of tungsten wires $0.25 \mathrm{~mm}$ in diameter were prepared by electrochemical etching in ambient conditions and substantially cleaned in UHV using direct current heating. Bias voltage is applied to the sample. The tuning fork tips used in NC-AFM/STM were made of platinum-iridium wires $0.05 \mathrm{~mm}$ in diameter cut by focused ion beam. The images have been presented using the WSXM software [20].

\section{RESULTS}

\section{A. Details of the adsorption geometry}

Large scale STM images of the $\operatorname{Ag}(111)$ and the $\mathrm{Cu}(111)$ surfaces after $\mathrm{Ru}(\mathrm{dbm})_{3}$ evaporation are shown in Figs. 2a and b. These images show that the two different objects already described in [18] on $\mathrm{Ag}(111)$ are the same on $\mathrm{Cu}(111)$. The most common structure consists in a two lobed entity (called type $B$ ) with an apparent height of about $4 \AA$ (insert in Fig 3 ).
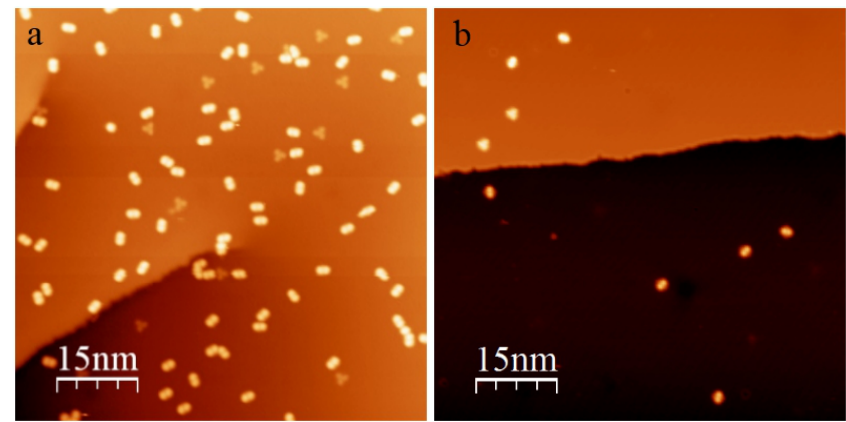

FIG. 2. STM image $75 \times 75 \mathrm{~nm}^{2}$ of the $\mathrm{Ru}(\mathrm{dbm})_{3}$ molecules after evaporation on (a) the $\mathrm{Ag}(111)$ and (b) $\mathrm{Cu}(111)$ substrate held at low temperature $\left(V_{s}=-1 \mathrm{~V}, I_{t}=1 \mathrm{pA}\right.$, $T=4.5 \mathrm{~K})$.

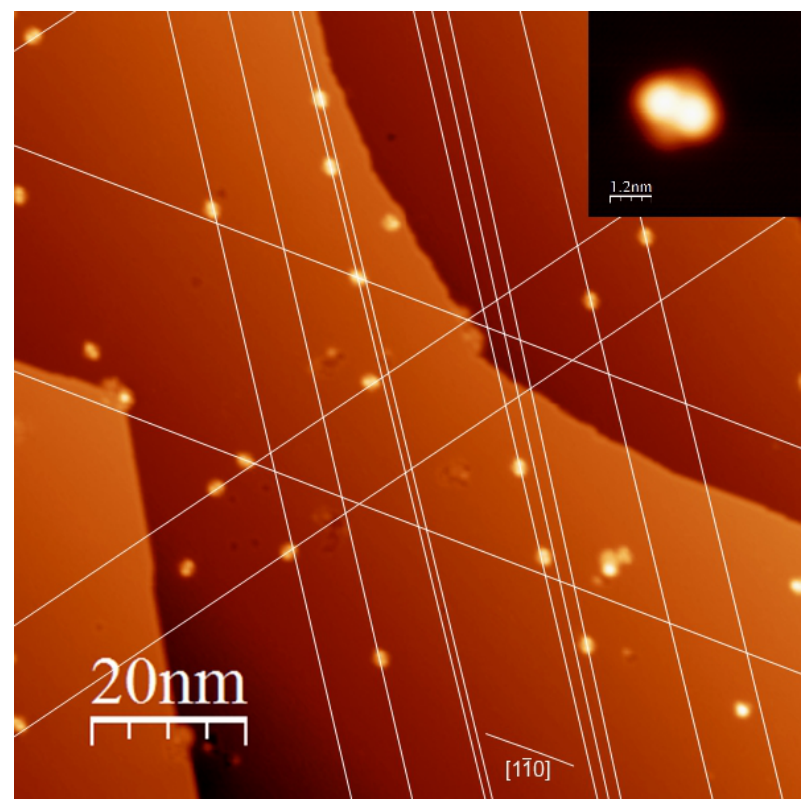

FIG. 3. Orientation of the bi-lobed structure on $\mathrm{Cu}(111)$. The orientation of the top ligand of most of the molecules are represented with white lines. Insert : Close up view $(5 \times 5$ $\left.\mathrm{nm}^{2}\right)$ of the bi-lobe structure called B. $\left(V_{s}=1.5 \mathrm{~V}, I_{t}=\right.$ $15 \mathrm{pA}, T=4.5 \mathrm{~K})$

The orientation of these bi-lobed structures with respect to the underlying structure is not randomly dis- 
tributed. To show this effect, white lines have been drawn in Fig. 3 along the direction of the bi-lobed shape molecules. We noticed that these lines are oriented along the three crystallographic $[1 \overline{1} 0]$ type directions of the sample. This observation is confirmed by the distance between two lines, which is always a multiple of the $\mathrm{Cu}$ lattice parameter, for example in Fig 3. This observation is also supported by the orientation of the molecule in the calculated images described in [18]. The same results are observed on the $\operatorname{Ag}(111)$ surface, which has a different lattice parameter than the $\mathrm{Cu}(111)$. This confirms that this effect is not due to the substrate but is an effect of the adsorption of the dbm ligands themselves along close packed directions.

The second type of object can be described as a threefold structure (called type $T$ ) presenting a lower apparent height of about $3 \AA$. Details of this structure are presented in Fig. 4 where small scale images performed on two of these objects are shown. These objects present two different orientations once adsorbed on the surface since the rotation of their peripheral groups is anticlockwise (Fig. 4a) or clockwise (Fig. 4b). Among the T structures, the right and left handed configurations are equally distributed on the surface. Note also that this geometry and the resulting chirality is the same whatever the substrate.

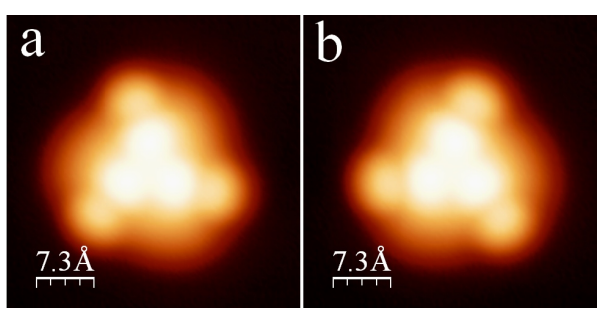

FIG. 4. Close up view $\left(3.6 \times 3.6 \mathrm{~nm}^{2}\right)$ of the second threefold structures called $\mathrm{T}$ type showing two eniantomers of the structure $\left(V_{s}=1.5 \mathrm{~V}, I_{t}=15 \mathrm{pA}, T=4.5 \mathrm{~K}\right)$.

The images presented so far show that the molecule adsorbs in two different ways on the surface. These results have been confirmed by molecular mechanics calculations in which the type B geometry corresponds to two dbm groups parallel to the surface and the $\mathrm{T}$ type to three phenyl groups forming an octahedral structure [18]. Therefore, the bi-lobed structure can be assimilated to a standing up form and the threefold structure to a laying down one. For clarity, these two forms are sketched in Fig. 5. Statistics show that the ratio is about $B / T=80 / 20$ between the two structures present on the surface, whatever the substrate, $\mathrm{Ag}(111)$ or $\mathrm{Cu}(111)$.

\section{B. STM manipulation}

To confirm that the two forms are produced by the same molecule, we show that it is possible to change its adsorption geometry or its appearance using the STM tip. Such manipulations are nevertheless tightly depen-
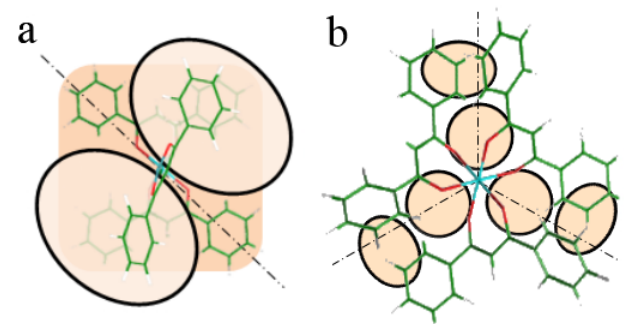

FIG. 5. Schema of the two adsorption geometries on the surface. The corresponding structure is added. a) Bi-lobed (B) and $\mathrm{b}$ ) threefold ( $\mathrm{T}$ ) form

dant on the tip shape. Details of these experiments are presented below.

\section{Adsorption geometry modification}

The first observed manipulation consists in "picking up" a threefold object (type $T$ ) in order to produce a bi-lobed conformation (type $B$ ). An example of this experiment is presented Fig.6.
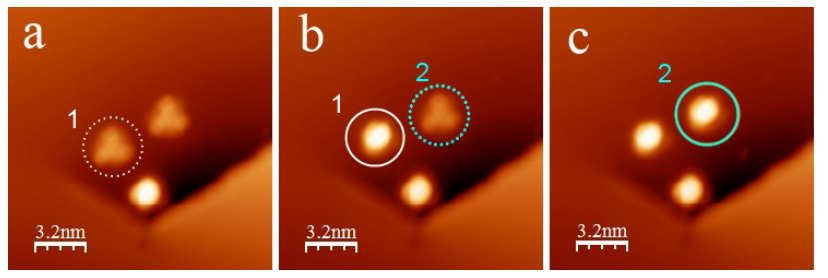

FIG. 6. STM images $15 \times 15 \mathrm{~nm}^{2}$ conducted during the modification of a type $T$ in a type $B$ structure: a) before manipulation 1 (dashed white circle), b) after manipulation 1 (white circle) and before manipulation 2 (blue dashed circle) and c) after modification 2 (full blue circle) $\left(V_{t}=1.5 \mathrm{~V}, I_{t}=15 \mathrm{pA}\right.$, $T=4.5 \mathrm{~K})$.

Fig. 6a shows a type $B$ and two type $T$ molecules. In a first step, the tip is placed above the white dash circled molecule (1). Next, the bias voltage is increased to a value of $2.5 \mathrm{~V}$, while the feedback loop maintains the tunneling current constant. This induces a sudden change in the molecular adsorption geometry to the bilobed conformation ( $B$ type) as seen in the white circle of Fig. $6 \mathrm{~b}$. The same procedure can be applied to the second $T$ molecule (blue dashed circle ). The tip is placed again above the considered molecule (2). After the rise of the bias voltage to $2.5 \mathrm{~V}$, the molecule geometry changes again to a type $B$ geometry (full blue circle in Fig. 6c). This process is reproducible only if the tip has a rather blunt shape. This phenomenon can be explained by a large area in the tip sample junction in which a high electric field is applied. By the way, the electrostatic force due to the presence of the electric field has an effect on the whole molecule, which induces a modification of the adsorption geometry instead of breaking bonds [21]. On 
the other hand, it is impossible to change the chirality of a $\mathrm{T}$ object whatever the sign and value of the applied voltage.

It is also impossible to obtain a threefold object ( $\mathrm{T}$ type) from a B form object. This is explained by the flat adsorption of two dbm moieties on the surface, which induces the formation of a very stable structure on the substrate. The last dbm points upward and has a larger degree of freedom. In this case, the high electric field breaks the chemical bonds instead of desorb the two other ligands. As a consequence, the molecule cannot lay down and does not exhibit a $\mathrm{T}$ type geometry.

\section{Switch manipulation}

Following the experiments reported before, it has been observed than sharper the tip, more unstable become the molecules when the bias voltage exceeds $+2 \mathrm{~V}$. This phenomenon is only observed on type $B$ molecules. Indeed, when a sharp tip is placed above a bi-lobed molecule and the bias voltage is raised up to about $2.1 \mathrm{~V}$, a slight change of the $B$ conformation is observed. The result of this manipulation is presented in Fig. 7a.
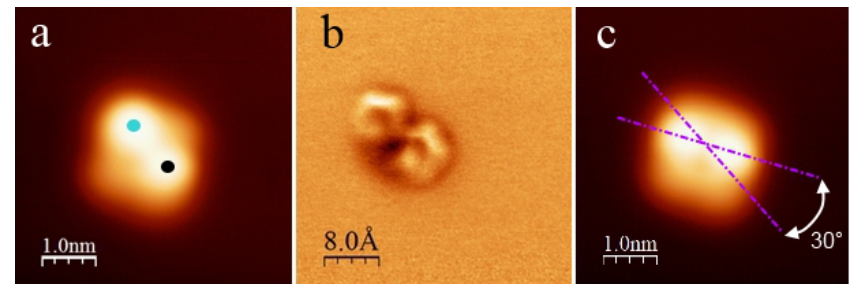

FIG. 7. a) STM image of a modified type $B$ molecule called "state 1" [The two marks on the molecule are discussed in the text] b) NC-AFM image of the same molecule in constant height mode $\left(4 \times 4 \mathrm{~nm}^{2}\right.$, and $V_{t}=200 \mathrm{mV}$, Amplitude $=$ $100 \mathrm{pm}, T=4.5 \mathrm{~K}) \mathrm{c}$ ) STM image of a modified type $B$ molecule called "state 2 ".

This image shows that the bi-lobe structure is still visible but with smaller lobes. Indeed, the apparent height only reaches $2.6 \AA$ against $3.8 \AA$ before the experiment. The square shape of the base made of two dbm is thus highlighted. This peculiar geometry called "state 1" in the following was investigated by non-contact AFM. As seen in Fig 7b, the NC-AFM image performed at constant height shows two cycles with four sides. It has been shown that the NC-AFM contrast strongly depends on the tip sample distance [22] and only the parts of the molecule within the same range of distance to the tip are resolved. Due to the three-dimensional geometry of the molecule and possible deformation of phenyl rings around the $\mathrm{Ru}$ center due to the electrical field, the upward dbm ligand is tilted with respect to the vertical. Note that such a NC-AFM contrast is never observed on unperturbed molecules since their upper dbm ligands always point strictly vertically. This two four sides cycles are therefore attributed to four of the six C-C bonds of the dbm ligand pointing upwards, the two others being closer to the $\mathrm{Ru}$ center. In addition, the ring diameter corresponds to the theoretical value. This image mostly shows that the molecule retains its structure after the bias voltage increases.

In the same experimental conditions, a second geometry is also observed. The top bi-lobe structure has rotated about $30^{\circ}$ and appears to be oriented along the side of the square base instead along the diagonal. This second geometry is called "state 2" and is presented in Fig.7c.

The modification of the bi-lobed form can be performed on the $\operatorname{Ag}(111)$ or $\mathrm{Cu}(111)$ surfaces if the tip is sharper enough. In addition, a switch between the two states can be observed. When the tip is located above one of the molecule lobes, the tip-surface distance variations can be recorded as a function of time, the tunneling current being kept constant. At low polarisation, the $\mathrm{z}$ tip position does not change, which means the molecule remains in its original state. When the bias voltage exceeds the threshold value of $+2.1 \mathrm{~V}$, the switch between the states 1 and 2 takes place, resulting in sudden jumps in the tip height.

Figs. $8 \mathrm{a}$ and $8 \mathrm{~b}$ illustrate this phenomenon. These curves conducted over several minutes at a bias voltage of $2.2 \mathrm{~V}$ show that the tip height is time dependent and presents two distinct levels. In a first experiment (Fig. 8a), a bias voltage of $2.2 \mathrm{~V}$ is applied on the bottom lobe in the state 1 (black circle in Fig. 7a). Then, the bi-lobed structure evolves to the state 2 (Fig 7c). The lobe is then out of the tip. As a consequence, state 1 corresponds to the high level of the graph, since state 2 corresponds to the low level. A calculation of the average residing time on each state shows the tip stays about $2.52 \pm 0.02$ seconds in state 1 and $1.32 \pm 0.02$ seconds in state 2. In a second experiment (Fig. 8b), the tip is placed above the blue circle of Fig. 7a. The tip height switches between state 1 where it is above the square base (low level) and state 2 where it is above a lobe of the bi-lobed structure (high level). The residing times are $1.7 \pm 0.02 \mathrm{sec}$ in state 2 and $5.6 \pm 0.02 \mathrm{sec}$ in state 1 respectively. The molecule preferentially adopts the low level state and then, the geometry presented in Fig. 7a. In both cases, the height variation is about $0.25 \AA$ and corresponds to the rotation of the upper dbm under the tip.

The life time of each state is random but the number of switches as a function of time depends on the bias voltage. To show this dependence, the tip is again located above the blue circle of Fig. 7 a but a bias voltage of $2.1 \mathrm{~V}$ is applied. Fig. $8 \mathrm{c}$ shows the variations of the tip height as a time function. The curve exhibits the same two steps shape but the time length of each state is longer than for an applied voltage of $2.2 \mathrm{~V}$. This value is about $20.25 \pm 0.02 \mathrm{sec}$ for the high level state against $9.15 \pm$ $0.02 \mathrm{sec}$ for the low level state. Therefore, the molecular conformations are more stable than at $2.2 \mathrm{~V}$, especially state 1 . This can be explain by the low electrical field, 

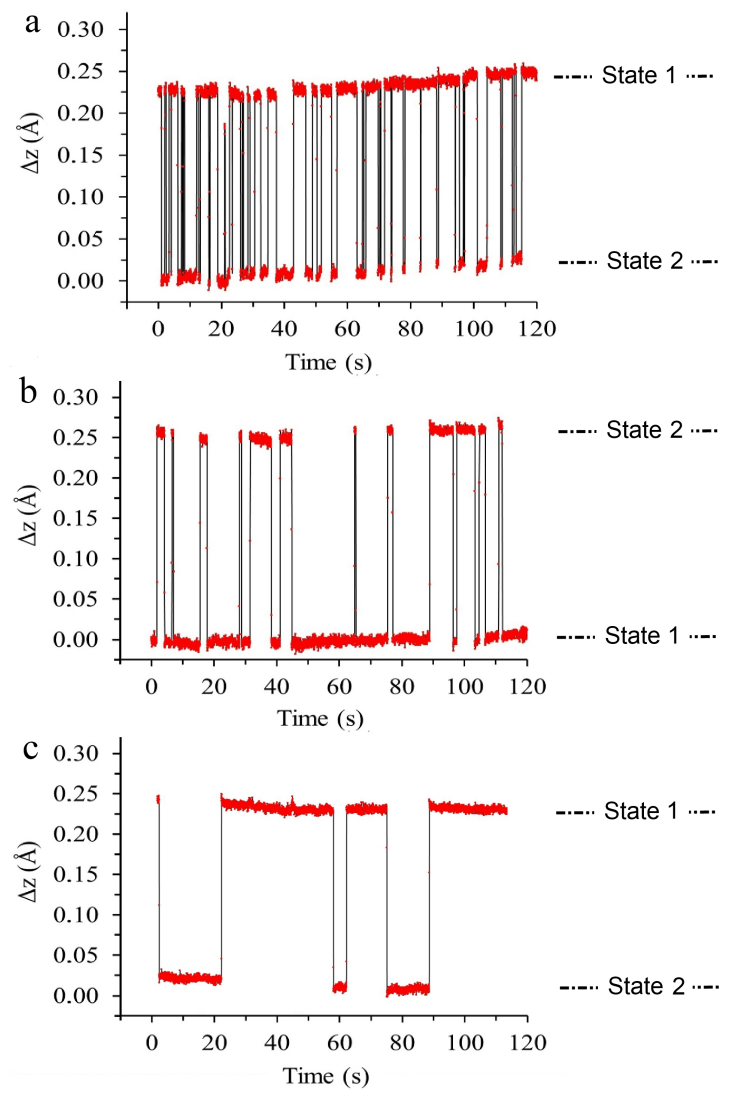

FIG. 8. Variations of the tip height as a function of time over a type $B$ structure at a bias voltage of $2.2 \mathrm{~V}$ while the tip is positioned above a) the black circle, b) the blue circle in Fig 7a. c) Variations of the tip height as a function of time at a bias voltage of $2.1 \mathrm{~V}$ while the tip is positioned above the black circle. These data have been extracted from a more complete data set recorded during $6 \mathrm{mn}$.

which can engage the switch but is not high enough to increase the transition rate. This phenomenon occurs for a very small bias range (from $2.1 \mathrm{~V}$ to $2.2 \mathrm{~V}$ since at $2.3 \mathrm{~V}$ the molecule is damaged) and does not depend on the tunneling current.

Using the variations of the tip height as a function of time, it is then possible to block with a high reproducibility the molecular configuration in its initial state (state 1 and initial state after adsorption) or in a new conformation (state 2). For this, the bias voltage is decreased below $2 \mathrm{~V}$ while the tip is on the chosen state. The tip scan is thus resumed and a new image is recorded to confirm the result of the manipulation.

\section{DISCUSSION}

Most of the experiments performed so far on mixed valence complexes deal with the observation of di-nuclear complexes deposited on metallic surfaces at relative high coverage $[4,5]$. The molecular interactions within the layer generally led to the formation of more or less self assembled domains in which the characterization of single molecules was rather difficult. However, the study of single moieties which can be further used to construct large molecules, appears fundamental. From this point of view, our experiments demonstrate that the $\mathrm{Ru}(\mathrm{dbm})_{3}$ complex can be easily evaporated using a standard technique to obtain isolated molecules on a metallic surface. The role of the dbm ligand is particularly interesting since it allows evaporation and adsorption on the surface without any damage to the molecule. This interest is also enhanced when one considers the two adsorption geometries of $\mathrm{Ru}(\mathrm{dbm})_{3}$. The capacity to access a bi-lobed form from a threefold geometry thanks to the STM tip shows these two configurations come from the same entire molecule and not a damaged one.

This particular property of dbm ligands is first underlined by the higher stability of the B form, where two $\mathrm{dbm}$ are lying flat on the substrate. Indeed, the adsorption energy of this latter was found to be $0.60 \mathrm{eV}$, while the $\mathrm{T}$ form only reaches $0.34 \mathrm{eV}$ on $\mathrm{Ag}(111)$ [18]. Thus, about $80 \%$ of the observed molecules present the $B$ form after adsorption. Moreover, these objects are oriented after adsorption along the three crystallographic [110] type directions of the sample. The same results observed on both $\mathrm{Cu}(111)$ and $\mathrm{Ag}(111)$ surfaces, which have different lattice parameters, confirm that this is due to the flat adsorption of the two dbm ligands on the substrate along close packed directions. In addition, it has been shown that a $\mathrm{T}$ form can be irreversibly "picked up" in order to produce a bi-lobed geometry. The irreversibility of the process, i.e. the impossibility to produce a $\mathrm{T}$ form from a bi-lobed geometry, confirms the higher stability of the molecule when the dbm ligands are adsorbed flat on the surface. All theses observations also suggest that the bi-lobe conformation will be more sensitive to the electrostatic field produced by the STM tip because of the dbm ligands pointing upwards. This sensitivity largely explains the presence of a molecular switch.

A molecular switch implies a reversible transformation between at least two distinct stable switching states, usually geometrical or valence isomers [23-26]. For example, M. Lastapis et al. selectively activated different reversible movements of an individual biphenyl molecule adsorbed on a $\mathrm{Si}(100)$ surface due to the application of a negative voltage of about $-3 \mathrm{~V}$ [27]. Similarly, L. Grill et al. observed the isomerization of azobenzene [28]. They showed that azobenzene adopts two different geometrical conformations: a planar (trans) and a non planar threedimensional (cis) form. The reversible switch is engaged by voltage pulses of about $6 \mathrm{~V}$ above a molecular island. Specific intra-molecular conformations or peculiar molecular adsorption geometries can also be achieved on a surface while they would not be present after evaporation, because they are energetically not favoured [29, 30]. Generally, these experiments are performed on small and/or flat molecules. These latter are easily manipulated or are particularly sensitive to the electric field locally produced 
by the STM tip to switch between different molecular states. For large bulky objects, these experiments are more difficult to perform and suffer from a lack of reproducibility. This can be partly explained by the relative fragility of such large structures.

Then, the ability to observe a reproducible switch on the $\mathrm{Ru}(\mathrm{dbm})_{3}$ molecule is particularly interesting. As said before, only the two lobed structure exhibits this characteristic, this adsorption geometry being particularly suited for this manipulation. The results show that a first step is yet required to engage the switching process. This involves applying a bias voltage above $+2 \mathrm{~V}$ above the molecule during a couple of seconds. A slight modification of the molecular appearance is then observed. The molecule reaches an "intermediate state" where the two lobes become slightly asymmetric and present a lower corrugation.

In this intermediate state, the ruthenium complex exhibits two distinct switching conformations which are voltage dependant: a square base adsorbed on the $\mathrm{Ag}(111)$ surface and a bi lobe structure pointing upwards with two distinct orientations (Fig.8). To switch between these two conformations, the tip is fixed above one of the two lobes and the positive bias voltage is increased (with a maximal value of $+2.2 \mathrm{~V}$ ). We noticed the molecule is stable at negative voltages down to $-3.5 \mathrm{~V}$ and the switch appears only for positive voltage from $+2.1 \mathrm{~V}$ (applied bias voltages below $-3.5 \mathrm{~V}$ or above $2.2 \mathrm{~V}$ destroy the molecule) Moreover, if the bias voltage is fixed just below the switch threshold and if the applied current is increased until $70 \mathrm{pA}$ (which is more than 10 times the initial value), the intermediate state is still stable. The only way to engage the molecular switch is to increase the bias voltage above $2 \mathrm{~V}$, independently of the current used. The switching rate only depends on the voltage value and not on the tunneling current (Figs. 8c and d.). This confirms that no inelastic effect comes into play in this process. The bias voltage range is rather small (from 2.1 to $2.2 \mathrm{~V}$ ), so that a statistical analysis is difficult to achieve. However, the different residing times shows that the state 1 is more stable.

The tip shape is also important to perform a given manipulation. If the tip is fine enough, these two conformations can switch in a controlled and reversible way. The modification is therefore closely linked to the localisation area of the electric field under the tip [31, 32]. Using a simple geometrical model of Coelho and Debeau [33], it is possible to calculate the lateral extension of the electrical field between a plane and an hyperbolic tip. Given the tunnelling currents and bias voltages used in these experiments, the tip-to-sample distance is estimated to be $\mathrm{d} \approx 1 \mathrm{~nm}$. Thus, a tip curvature radius of $8 \AA$ implies that the area around the tip apex in which the field is between $E_{\max }=V_{t} / d$ and $75 \%$ of $E_{\max }$ corresponds to the size of the molecule (diameter of $1.48 \mathrm{~nm}$, Fig. 1). This area is more than 3 times larger $\left(6.2 \mathrm{~nm}^{2}\right)$ when the curvature radius is only $12 \AA$. Then, the tip has to be terminated by a single atom to perform the switch ex- periments on the B state (the field is located on a precise point of the molecule). For a blunt tip made of an atomic cluster fixed on a macroscopic base, the lateral extension of the field is used to manipulate the whole $\mathrm{T}$ molecule to produce a B structure or to cause eventually a lateral displacement of this latter. As a consequence, the B form does not evolve to the intermediate state and no switch is observed.

The switching from $\mathrm{T}$ to $\mathrm{B}$ type occurs at a bias voltage of $2.5 \mathrm{~V}$, i.e. a value higher than the threshold for dissociation of a B molecule $(2.3 \mathrm{~V})$. This difference can also be explained by an effect of the electric field in the STM junction. On T objects, the corrugation is about $3 \AA$ and the adsorption geometry is relatively flat (three dbm lying on the surface, Fig. 5b). For B type molecules, the apparent height is $4 \AA$ but a dbm group points upwards. The first adsorption calculations show that the two structures present a height difference of about $2.5 \AA$ [18]. This implies that the molecule-tip distance is larger on a $\mathrm{T}$ type than on a B structure, resulting in a lower electric field. A T type molecule is then able to switch in a B type at $2.5 \mathrm{~V}$ while a $\mathrm{B}$ type would be dissociated at the same voltage.

Another point to consider is the irreversibility of the process. Indeed, the switched molecule never returns to the original bi-lobed shape presenting the highest corrugation, whatever the value and sign of the applied bias voltage (this latter being smaller than $2.3 \mathrm{~V}$ to avoid fragmentation). A first hypothesis suggests that the applied bias voltage could give to the molecule enough energy to adopt a new adsorption geometry as the substrate is held at $4 \mathrm{~K}$ during evaporation. From this point of view, $\mathrm{Ru}(\mathrm{dbm})_{3}$ are relatively fragile as compared to planar molecules which can be imaged at voltages above $3 \mathrm{~V}$ without any problem. Then, the change in geometry is probably not sufficient to explain the irreversibility of the switching process. It was then assumed that the first step necessary to allow the switch is accompanied by a modification of the oxidation degree of the $\mathrm{Ru}$ atom. Indeed, this atom can change its oxidation state from $\mathrm{Ru}(\mathrm{III})$ to $\mathrm{Ru}(\mathrm{II})$ due to electron injection induced by the STM tip. This effect has already been observed on $\mathrm{Cu}$ complexes. Indeed, Leoni et al. reported the switching behaviour of a $\mathrm{Cu}(\mathrm{dbm})_{2}$ complex where the transition between a square planar and a 3D tetrahedral geometry was due to the transition between the $\mathrm{Cu}(\mathrm{II})$ and $\mathrm{Cu}(\mathrm{I})$ oxidation states [30]. However, as opposed to our experimental results, their process is perfectly reversible. In the $\mathrm{Ru}(\mathrm{dbm})_{3}$ case, it is unlikely that the negative charge irreversibly remains on the ruthenium, which discards this hypothesis.

As explained before, the process is driven by the electric field in the STM junction. The electric field can induce deformation for permanently charged atom or molecules [34]. It can also interact with dipolar moments of certain molecules [16]. The $\mathrm{Ru}(\mathrm{III})$ ion exhibits an octahedral low spin $\mathrm{d}^{5}$ configuration. The degenerate 


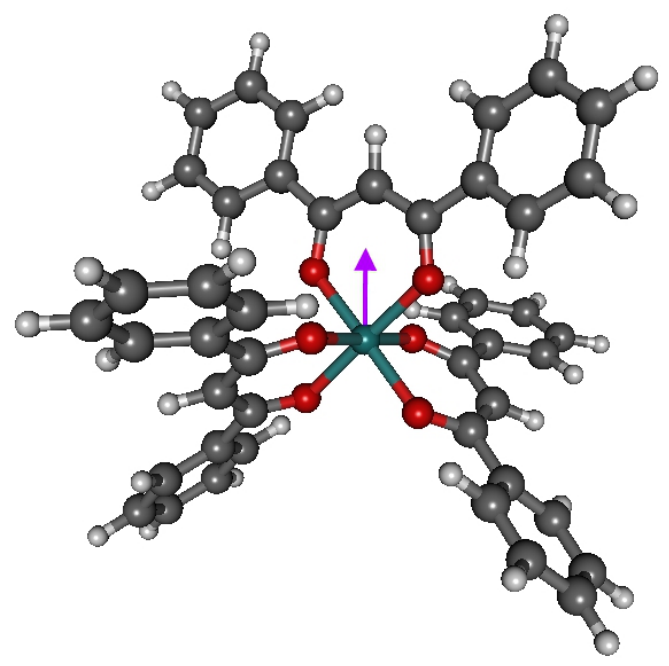

FIG. 9. Structure obtained by DFT calculations in gaz phase showing the dipole moment orientation (purple arrow). A value of $0.98 \mathrm{D}$ has been obtained.

ground-state leads to a break in degeneracy which stabilizes the molecule and reduces its symmetry according to the Jahn-Teller effect [35]. In a previous paper (supplementary data in [18]), it was noticed that two Ru-O bond lengths in the $\mathrm{Ru}(\mathrm{dbm})_{3}$ compound are slightly longer than the four others (about $0.03 \AA$ ). A dipole moment of $0.98 D$ has been also calculated for the compound in gas phase using DFT calculations [36]. As seen in Fig 9, this dipole moment takes place between the $\mathrm{Ru}$ atom and one of the dbm.

After adsorption, the B type molecule is even more distorted due to the interaction with the surface and its dipole moment should increase. Moreover, the AFM image shows that in the intermediate state, the upper dbm ligand is tilted compared to the original bi-lobed shape (Fig.7b). This also involves a tilt of the dipole moment. If this moment is not collinear to the electric field created by the tip, the state becomes unstable and sets off a rotational movement of the upper lobe limited by the steric hindrance within the molecule. The rotation of the whole upper dbm ligand can be also accompanied by a small rotation of each phenyl group. Indeed, the rotation of these later requires only a few tenths of eV [37]. Sim- ple molecular dynamics calculations demonstrate that a rotation of about 20 degrees of the upper dbm moiety is possible. This movement also includes a small rotation of the two phenyls of a few degrees in good agreement with the hypothesis previously discussed and with the NC-AFM observations. The effect of the electrical field on the molecular dipole moment is linked to the presence of the so-called intermediate state which in turn implies a molecular structure modification. It is assumed that this intermediate state is related to the dissociation of one of the top $\mathrm{C}-\mathrm{H}$ bonds of the molecule. Controlled breaking of $\mathrm{C}-\mathrm{H}$ bonds have already been conducted using an STM tip [38, 39]. Particularly, Ho et al. have broken $\mathrm{C}-\mathrm{H}$ bonds on different molecules and observed that the $\mathrm{H}$ atom was imaged such as a small depression on a copper surface. This is good agreement with the small depression observed in the molecule center in state 2 . The dehydrogenation can also explain that the bi-lobed geometry needs a positive voltage and a sharp tip to access the "intermediate state" and is also consistent with the irreversibility of the phenomenon.

\section{SUMMARY}

It has been observed that tris(dibenzoylmethanato)ruthenium complex adsorbs in two different ways on $\mathrm{Ag}(111)$ and $\mathrm{Cu}(111)$ surfaces: a bi-lobed and a chiral threefold structure. We have shown that the threefold structure can stand up to adopt a bi-lobed conformation, in which the upper ligand and then, all the molecule is oriented along a dense crystallographic direction. This last structure can irreversibly adopt a new conformation which is able to switch between two geometries. The switch itself is caused by an applied bias voltage from $2.1 \mathrm{~V}$. These results are consistent with a first dehydrogenation of the molecule followed by a tilted dipole moment sensitive to the local electrical field produced by the tip. Calculations are in hand to solve the very interesting but also complex phenomenon. Finally, the results show that it is possible to manipulate large 3D objects, which is very promising for future molecular electronic devices made of mixed valence complexes with several ruthenium centers.
[1] C. Joachim, J. K. Gimzewski, A. Aviram Nature 408 (2000) 541.

[2] N. Koch, S. Duhm, J. P. Rabe Phys. Rev. Lett. 95 (2005) 237601.

[3] F. Jackel, U. G. E. Perera, V. Iancu, K. F. Braun, N. Koch, J. P. Rabe, S. W. Hla, Phys. Rev. Lett. 100 (2008) 126102.

[4] Y. Lu, R. C. Quardokus, C. S. Lent, F. Justaud, C. Lapinte, S. A. Kandel, J. Am. Chem. Soc. 132 (2010) 13519.
[5] R. C. Quardokus, Y. Lu, N. A. Wasio, C.S. Lent, F. Justaud, C. Lapinte, S.A. Kandel, J. Am. Chem. Soc. 134 (2012) 1710.

[6] J.V. Barth, Ann. Rev. Phys. Chem. 58 (2007) 375.

[7] Ch. Nacci, J. Lagoute, X. Liu, S. Fölsch, Appl. Phys. A 93 (2008) 313.

[8] S. Info, Y. Cao, K.S. Yong, Z.Q. Wang, W.S. Chin, Y.H. Lai, J.F. Deng, G.Q. Xu, J. Am. Chem. Soc. 122 (2000) 1812. 
[9] J. Repp, G. Meyer, S.M. Stojkovic, A. Gourdon, C. Joachim, Phys. Rev. Lett. 94 (2005) 26803.

[10] S.E. Grillo, H. Tang, C. Coudret, S. Gauthier, Chem. Phys. Lett. 355 (2002) 289.

[11] B. Calmettes, S. Nagarajan, A. Gourdon, M. Abel, L. Porte, R. Coratger, Angew. Chem. Int. 47 (2008) 6994.

[12] S.J. van der Molen, P. Liljeroth, J. Phys. Condens. Matter 22 (2010) 133001.

[13] P. Liljeroth, J. Repp, G. Meyer, Science 317 (2007) 1203

[14] M. Alemani, S. Selvanathan, F. Ample, M. V. Peters, K.H. Rieder, F. Moresco, C. Joachim, S. Hecht, L. Grill, J. Phys. Chem. C 112 (2008) 10509.

[15] C. Girard, C. Joachim, C. Chavy, P. Sautet, Surf. Sci. 282 (1993) 400.

[16] L. Grill, K.H. Rieder, F. Moresco, S. Stojkovic, A. Gourdon, C. Joachim, Nano Lett. 6 (2006) 2685.

[17] Y.L. Huang, Y. Lu, H. Huang, S. Kera, N. Ueno, W. Chen, Small 8 (2012) 1423.

[18] S. Munery, N. Ratel-Ramond, Y. Benjalal, L. Vernisse, O. Guillermet, X. Bouju, R. Coratger, J. Bonvoisin, Eur. J. Inorg. Chem. 17 (2011) 2698.

[19] L. Vernisse, S. Munery, N. Ratel-Ramond, Y. Benjalal, O. Guillermet, X. Bouju, R. Coratger, J. Bonvoisin, J. Phys. Chem. C 116 (2012) 13715.

[20] I. Horcas, R. Fernandez, J. M. Gomez-Rodriguez, J. Gomez-Herrero, A. M. Baro, Rev. Sci. Instrum. 78 (2007) 013705.

[21] C. Chapman and I. Paci, J. Phys. Chem. C 114 (2010) 20556.

[22] L. Gross, F. Mohn, N. Moll, P. Liljeroth, G.Meyer, Science 325 (2009) 1110.

[23] S. Hla, JVST B 23(4) (2004) 1351.
[24] N. Lorente, R. Rurali, H. Tang, J. Phys.: Condens. Matter 17 (13) (2005) 1049.

[25] F. Moresco, G. Meyer, K.-H. Rieder, H. Tang, A. Gourdon, C. Joachim, Phys. Rev. Lett. 86 (2001) 672.

[26] B.-Y. Choi, S.-J. Kahng, S. Kim, H. Kim, H. W. Kim, Y. J. Song, J. Ihm, Y. Kuk, Phys. Rev. Lett. 96 (2006) 156106.

[27] M. Lastapis, M. Martin, D. Riedel, L. Hellner, G. Comtet, G. Dujardin, Science 5724 (2005) 1000.

[28] M. Alemani, M. V. Peters, S. Hecht, K.-H. Rieder, F. Moresco, L. Grill, J. Am. Chem. Soc. 128, (2006) 14446.

[29] B. L. Ferringa, Molecular Switches (Wiley-VCH, Weinheim, 2001)

[30] T. Leoni, O. Guillermet, H. Walch, V. Langlais, A. Scheuermann, J. Bonvoisin, S. Gauthier, Phys. Rev. Lett. 106 (2011) 216103.

[31] G. Füchsel, T. Klamroth, J. Dokić, P. Saalfrank, J. Phys. Chem. B 110(33) (2006) 16337.

[32] L. Grill, K.-H. Rieder, F. Moresco, S. Stojkovic, A. Gourdon, C. Joachim, Nano Lett. 5 (2005) 859.

[33] R. Coelho and J. Debeau, J. Phys. D: Appl. Phys. 4 (1970) 1266.

[34] X. H. Qiu, G.V. Nazin, and W. Ho, Phys. Rev. Lett. 93 (2004) 196806.

[35] H. A. Jahn and E. Teller, Proc. R. Soc. London A 161(1937) 220.

[36] J. Bonvoisin and I. Ciofini, Dalton Trans. 42 (2013) 7943.

[37] N. Henningsen, K. J. Franke, I. F. Torrente, G. Schulze, B. Priewisch, K. Rück-Braun, J. Dokic̀, T. Klamroth, P. Saalfrank, and J. I. Pascual, J. Phys. Chem. C 111 (2007) 14843.

[38] L.J. Lauhon, W. Ho, Surf. Sci. 451 (2000) 219.

[39] Y. Kitaguchi, A. Shiotari, H. Okuyama, S. Hatta, and T. Aruga, J. Chem. Phys. 134 (2011) 174703. 